\title{
Electromagnetic Transition Form Factors of Light Mesons
}

\author{
Pieter Maris f $^{2}$ \\ Department of Physics, North Carolina State University, Raleigh, NC 27695-8202 \\ Peter C. Tandy \\ Department of Physics, Center for Nuclear Research, Kent State University, Kent, OH 44240
}

(Dated: October 9, 2018)

\begin{abstract}
We study selected meson transition processes and associated form factors within a model of QCD based on the Dyson-Schwinger equations truncated to ladder-rainbow level. The infrared structure of the ladder-rainbow kernel is described by two parameters; the ultraviolet behavior is fixed by the one-loop renormalization group behavior of QCD. The work is restricted to the $u$ and $d$ quark sector and allows a Poincaré-covariant study of the radiative decays: $\rho \rightarrow \pi \gamma, \omega \rightarrow \pi \gamma$, and $\pi^{0} \rightarrow \gamma \gamma$. Particular attention is paid to the form factors for the associated transitions: $\gamma^{*} \pi^{0} \rightarrow \gamma$, $\gamma^{*} \pi^{0} \rightarrow \gamma^{*}, \gamma^{*} \pi \rightarrow \rho$ and $\gamma^{*} \rho \rightarrow \pi$. The latter two processes are of interest as contributors to meson electroproduction from hadronic targets away from the $s$-channel resonance region. We use the present QCD model to explore limitations to the assumption that coupling to a $t$-channel virtual $\bar{q} q$ correlation can be modeled as meson exchange.
\end{abstract}

PACS numbers: Pacs Numbers: 24.85.+p, 14.40.-n, 13.40.-f, 11.10.St, 12.38.Lg

\section{INTRODUCTION}

At a soft scale, the electromagnetic content of hadrons reveals the distribution of current generated by the interacting constituent dressed quarks. This useful insight into non-perturbative hadron dynamics is expressed in terms of electromagnetic form factors obtained from exclusive processes such as elastic lepton scattering or leptonic transitions to specific hadronic states. At a hard scale, typified by present deep inelastic scattering experiments, much of the final state non-perturbative dynamics is reflected in the initial distributions of quark and gluon partons which are probed perturbatively. The unraveling of this information from structure functions will require a connection with non-perturbative QCD calculations and models. Although some lattice QCD studies have begun to produce moments of structure functions [1, 2], the opportunities are very limited at present. Useful quantities for calibration of models of QCD are the electromagnetic elastic and transition form factors of hadrons.

The most extensive hadronic models were designed to study the mass spectrum and decays and often contain elements that limit their use for developing electroweak form factors. Examples include: non-relativistic kinematics, a lack of manifest Poincaré covariance, no quark sea, no dynamical gluons, no QCD renormalization group behavior for evolution of scale, and no confinement of quarks. Covariant relativistic field theory models that are simpler than QCD, but respect dynamical chiral symmetry breaking, have long been utilized for the modeling of soft physics such as the hadronic mass spectrum. For example, such progress has been reviewed within the

\footnotetext{
* pmaris@unitv.ncsu.edu
}

t tandy@cnr2.kent.edu
Nambu-Jona-Lasinio model [3] and the Global Colour Model [4, 5. In the case of the latter, soft electromagnetic form factors of mesons and meson transitions have been considered [5]. For hard electromagnetic processes, studies of deep inelastic scattering within the NambuJona-Lasinio model [6, 7] have helped clarify some of the issues confronting work within a quark field theory format.

Here we summarize recent progress in the soft QCD modeling of light meson electromagnetic transition form factors based on the set of Dyson-Schwinger equations [DSEs] of the theory [8, 9]. The model we apply here has been previously shown to give an efficient description of the masses and electroweak decays of the light pseudoscalar and vector mesons 10, 11. Here we work in the $u$ and $d$ quark sector and treat electromagnetic processes involving $\pi, \rho$ and $\omega$. This covariant approach accommodates quark confinement and implements the QCD one-loop renormalization group behavior. The performance of this model for deep inelastic scattering phenomena can be gauged from that of a simplified version that has recently produced excellent results for the pion valence quark distribution amplitude [12].

An issue that arises in studies of electromagnetic interactions with hadrons is vector meson dominance [VMD]. Within a quark-gluon model that dynamically produces the vector meson pole in the dressed photon-quark vertex, the validity and effectiveness of extrapolating such a mechanism to nearby momentum domains can be tested. The pion charge radius and low- $Q^{2}$ charge form factor have recently been explored from that perspective [13. The empirical effectiveness of the simple VMD assumption in that case is much greater than its faithfulness to the underlying dynamics. A similar example is found here in the transition $\gamma^{*} \pi^{0} \rightarrow \gamma$. The vector meson resonance pole term extrapolated to the photon point produces an estimate of the $\rho$ radiative decay coupling con- 
stant $g_{\rho \pi \gamma}$ in terms of the $\pi$ decay coupling constant $g_{\pi \gamma \gamma}$ that is accurate to within a few percent. For a large range of spacelike momentum, we find the shape of the form factor for this transition to be consistent with a monopole with mass scale $m_{\rho}$; this is also consistent with analyses of the asymptotic behavior.

When a process like the above involves a $\bar{q} q$ correlation with a spacelike invariant mass, there arises the question of how accurately it can be modeled by meson exchange when the meson is a bound state defined at a unique (timelike) invariant mass. This question is here brought into sharper focus by examination of form factors for transition processes $\gamma^{*} M M^{\prime}$ that arise in meson exchange models [14. Here $M$ is a virtual $t$-channel $\bar{q} q$ correlation such as might be provided by a hadronic target, and $M^{\prime}$ is a physical produced meson. Our model of the underlying quark-gluon dynamics is used to investigate the extent to which the virtuality of $M$ influences the $\gamma^{*} M M^{\prime}$ form factor that should be employed in meson exchange models, given that the employed $t$-channel propagator for $M$ is of the standard point meson type. We consider the cases where $M$ can be vector or pseudoscalar. The domain of accuracy of the meson exchange assumption is explored.

In Sec. II we use the generic coupling of a pseudoscalar bosonic object to a pair of vector bosons in impulse approximation to define our notation. There we introduce the required dressed quark propagator from the QCD quark DSE in rainbow approximation, and the required vertex amplitudes from the Bethe-Salpeter equation [BSE] in ladder approximation. We also outline there the phenomenological infrared content and parameterization of the model and summarize the resulting pseudoscalar and vector meson properties. We specialize to the $\pi \gamma \gamma$ process in Sec. III, discussing both the coupling constant and two different transition form factors and their asymptotic behavior. In Sec. IV the $\rho \pi \gamma$ coupling is discussed from three different aspects: the coupling constants and widths for radiative decay of $\rho$ and $\omega$, the form factor for the $\gamma^{*} \pi \rightarrow \rho$ transition, and the form factors for $\gamma^{*} P \rightarrow \rho$ and $\gamma^{*} V \rightarrow \pi$ where $P$ and $V$ are virtual $\bar{q} q$ objects having the quantum numbers of ground state pseudoscalar and vector mesons respectively. Here the connection to $t$-channel meson exchange is explored. A summary can be found in Sec. V.

\section{PSEUDOSCALAR-VECTOR-VECTOR PROCESSES IN IMPULSE APPROXIMATION}

Here we analyse pseudoscalar-vector-vector processes (PVV) characterized by a generic PVV vertex that, in QCD in impulse approximation, is described by the quark loop integral

$$
\begin{aligned}
\Lambda_{\mu \nu}^{P V V}(P ; Q)= & N_{c} \int_{q}^{\Lambda} \operatorname{Tr}\left[S^{a}\left(q_{a}\right) \Gamma_{P}^{a \bar{b}}\left(q_{a}, q_{b}\right) S^{b}\left(q_{b}\right)\right. \\
& \left.\times \Gamma_{\mu}^{b \bar{c}}\left(q_{b}, q_{c}\right) S^{c}\left(q_{c}\right) \Gamma_{\nu}^{c \bar{a}}\left(q_{c}, q_{a}\right)\right]
\end{aligned}
$$

Here $\Gamma_{P}^{a \bar{b}}\left(q_{a}, q_{b}\right)$ is the pseudoscalar vertex function for a pseudoscalar coupling to an outgoing quark with momentum $q_{a}$ and flavor $a=u, d$, and an incoming quark with momentum $q_{b}$ and flavor $b=u, d$; similar definitions hold for the two vector vertex functions $\Gamma_{\mu}$ and $\Gamma_{\nu}$. The external momenta are $Q=q_{c}-q_{a}$ and $P=q_{b}-q_{c}$ for the vector vertices, and $K=-(P+Q)=q_{b}-q_{a}$ for the pseudoscalar vertex, all flowing into the triangle diagram.

The notation $\int_{q}^{\Lambda} \equiv \int^{\Lambda} d^{4} q /(2 \pi)^{4}$ stands for a translationally invariant regularization of the integral, with $\Lambda$ being the regularization mass-scale. The regularization can be removed at the end of all calculations, by taking the limit $\Lambda \rightarrow \infty$. It is understood that the same regularization is applied to the calculated dressed quark propagators $S(q)$ and vertex functions $\Gamma$ appearing in Eq. (11), and that the quark propagators are renormalized at a convenient spacelike momentum scale before taking the limit $\Lambda \rightarrow \infty$.

This generic PVV vertex can be specialized in an obvious way to such processes as the $\pi^{0} \rightarrow \gamma \gamma$ decay, radiative decays such as $\rho \rightarrow \pi \gamma$, and transitions such as $\gamma^{*} \pi \rightarrow \rho$. In general, one has to add loop integrals corresponding to the different orderings of the vertices and the various flavor-labeled components of neutral mesons, in order to get the vertex describing an actual physical process.

The form factor associated with the generic PVV vertex is identified from the general form

$$
\begin{aligned}
\Lambda_{\mu \nu}^{P V V}(P ; Q)= & C F_{P V V}\left((P+Q)^{2}, P^{2}, Q^{2}\right) \\
& \times \epsilon_{\mu \nu \rho \sigma} P_{\rho} Q_{\sigma}
\end{aligned}
$$

normalized in such a way that $F_{P V V}=1$ for on-shell external momenta; the constant $C$ contains the coupling constant, together with numerical factors such as isospin, symmetry factors, factors of $\pi$ from the integration measure and so on. This will be made clear for specific cases discussed later. Note that from the three external momenta $(P, Q, K)$, two of which are independent, there are several choices for the independent Lorentz scalar quantities that a form factor depends on. The choice of form factor variables will be dictated by the process in question. The form in Eq. (2) facilitates a study of the dependence upon the momenta of the vector objects. To study the dependence upon the momentum $K=-(P+Q)$ of the pseudoscalar object, we would take advantage of the antisymmetry of the $\epsilon$ tensor to use

$$
\begin{aligned}
\Lambda_{\mu \nu}^{P V V}(-(Q+K) ; Q)= & C F_{P V V}\left(K^{2},(K+Q)^{2}, Q^{2}\right) \\
& \times \epsilon_{\mu \nu \rho \sigma} Q_{\rho} K_{\sigma} .
\end{aligned}
$$

For electromagnetic interactions, electromagnetic current conservation is manifest if the approximations used for the dressing of the quark propagators, meson BetheSalpeter amplitudes [BSAs], and the quark-photon vertices are dynamically consistent with the approximation used for the photon-hadron interaction. Rainbow-ladder truncation of the DSE and BSE, in combination with impulse approximation for the photon-meson coupling satisfies this consistency requirement [15]. 


\section{A. Dyson-Schwinger Equations}

The DSE for the renormalized quark propagator in Euclidean space is

$$
\begin{aligned}
S(p)^{-1}= & i Z_{2} \not p+Z_{4} m_{q}(\mu)+ \\
& Z_{1} \int_{q}^{\Lambda} g^{2} D_{\mu \nu}(k) \frac{\lambda^{a}}{2} \gamma_{\mu} S(q) \Gamma_{\nu}^{a}(q, p),
\end{aligned}
$$

where $D_{\mu \nu}(k)$ is the dressed-gluon propagator, $\Gamma_{\nu}^{a}(q, p)$ the dressed-quark-gluon vertex, and $k=p-q$. The most general solution of Eq. (1) has the form $S(p)^{-1}=i p A\left(p^{2}\right)+B\left(p^{2}\right)$ and is renormalized at spacelike $\mu^{2}$ according to $A\left(\mu^{2}\right)=1$ and $B\left(\mu^{2}\right)=m_{q}(\mu)$ with $m_{q}(\mu)$ being the current quark mass. We use the Euclidean metric where $\left\{\gamma_{\mu}, \gamma_{\nu}\right\}=2 \delta_{\mu \nu}, \gamma_{\mu}^{\dagger}=\gamma_{\mu}$ and $a \cdot b=\sum_{i=1}^{4} a_{i} b_{i}$.

Mesons can be studied by solving the homogeneous BSE for $q^{a} \bar{q}^{b}$ bound states

$$
\begin{aligned}
\Gamma_{H}^{a \bar{b}}\left(p_{+}, p_{-}\right)= & \int_{q}^{\Lambda} K(p, q ; P) \\
& \otimes S^{a}\left(q_{+}\right) \Gamma_{H}^{a \bar{b}}\left(q_{+}, q_{-}\right) S^{b}\left(q_{-}\right),
\end{aligned}
$$

where $a$ and $b$ are flavor indices, $p_{+}=p+\eta P$ and $p_{-}=p-(1-\eta) P$ are the outgoing and incoming quark momenta respectively, and $q_{ \pm}$is defined similarly. The kernel $K$ is the renormalized, amputated $q \bar{q}$ scattering kernel that is irreducible with respect to a pair of $q \bar{q}$ lines. This equation has solutions at discrete values of $P^{2}=-m_{H}^{2}$, where $m_{H}$ is the meson mass. Together with the canonical normalization condition for $q \bar{q}$ bound states, it completely determines $\Gamma_{H}$, the bound state BSA. The different types of mesons, such as pseudoscalar, vector, etc are characterized by different Dirac structures. The most general decomposition for pseudoscalar bound states is 10

$$
\begin{aligned}
\Gamma_{P S}\left(q_{+}, q_{-}\right)= & \gamma_{5}\left[i E\left(q^{2} ; q \cdot P ; \eta\right)\right. \\
& +\not P F\left(q^{2} ; q \cdot P ; \eta\right)+\not k G\left(q^{2} ; q \cdot P ; \eta\right) \\
& \left.+\sigma_{\mu \nu} P_{\mu} q_{\nu} H\left(q^{2} ; q \cdot P ; \eta\right)\right]
\end{aligned}
$$

where the invariant amplitudes $E, F, G$ and $H$ are Lorentz scalar functions of $q^{2}$ and $q \cdot P$. For charge eigenstates, these amplitudes are appropriately odd or even in the charge parity odd quantity $q \cdot P$. In the case of the $0^{-+}$pion, for example, the amplitude $G$ is odd in $q \cdot P$, the others are even. Note also that these amplitudes explicitly depend on the momentum partitioning parameter $\eta$. However, so long as Poincaré invariance is respected, the resulting physical observables are independent of this parameter 15.

The meson BSA $\Gamma^{a \bar{b}}$ is normalized according to the canonical normalization condition

$$
\begin{aligned}
2 P_{\mu}= & N_{c} \frac{\partial}{\partial P_{\mu}} \operatorname{Tr}\left\{\int_{q}^{\Lambda} \bar{\Gamma}^{b \bar{a}}\left(\tilde{q}^{\prime}, \tilde{q}\right) S^{a}\left(q_{+}\right) \Gamma^{a \bar{b}}\left(\tilde{q}, \tilde{q}^{\prime}\right) S^{b}\left(q_{-}\right)\right. \\
& \left.+\int_{k, q}^{\Lambda} \bar{\chi}^{b \bar{a}}\left(\tilde{k}^{\prime}, \tilde{k}\right) K(k, q ; P) \chi^{a \bar{b}}\left(\tilde{q}, \tilde{q}^{\prime}\right)\right\}
\end{aligned}
$$

at $P^{2}=Q^{2}=-m_{H}^{2}$, with $\tilde{q}=q+\eta Q, \tilde{q}^{\prime}=q-(1-\eta) Q$, and similarly for $\tilde{k}$ and $\tilde{k}^{\prime}$. For vector mesons, it is understood that one must contract and average over the Lorentz indices of the (transverse) BSAs to account for the three independent polarizations.

We will later need the following exact expression for the pion decay constant $f_{\pi}$ :

$$
f_{\pi} P_{\mu}=Z_{2} N_{c} \int_{q}^{\Lambda} \operatorname{Tr}\left[\gamma_{5} \gamma_{\mu} S\left(q_{+}\right) \Gamma_{\pi}\left(q_{+}, q_{-}\right) S\left(q_{-}\right)\right] .
$$

Since a massive vector meson bound state is transverse, the BSA requires eight covariants for its representation. We choose the transverse projection of the form

$$
\begin{aligned}
\Gamma_{\mu}\left(q_{+}, q_{-}\right)= & \gamma_{\mu} V_{1}+q_{\mu} \not 1 V_{2}+q_{\mu} \not P V_{3} \\
& +\gamma_{5} \epsilon_{\mu \alpha \nu \beta} \gamma_{\alpha} q_{\nu} P_{\beta} V_{4}+q_{\mu} V_{5} \\
& +\sigma_{\mu \nu} q_{\nu} V_{6}+\sigma_{\mu \nu} P_{\nu} V_{7} \\
& +q_{\mu} \sigma_{\alpha \beta} q_{\alpha} P_{\beta} V_{8} .
\end{aligned}
$$

This form is a variation of that used in Ref. [11 that is simpler and easier to use in many respects. The invariant amplitudes $V_{i}$ are Lorentz scalar functions of $q^{2}$ and $q \cdot P$ and again, for charge eigenstates, they are either odd or even in $q \cdot P$. For the $1^{--} \rho$ meson, $V_{3}$ and $V_{6}$ are odd, the other amplitudes are even.

The quark-photon vertex is $\tilde{\Gamma}_{\mu}^{a}=\hat{Q}^{a} \Gamma_{\mu}^{a}$ where $\hat{Q}^{a}$ is the $a$-quark electric charge and the amplitude $\Gamma_{\mu}^{a}$ is normalized so that its bare (UV) limit is $\gamma_{\mu}$. The vector vertex $\Gamma_{\mu}^{a}$ with total momentum $Q=p_{+}-p_{-}$satisfies the inhomogeneous BSE

$$
\begin{aligned}
\Gamma_{\mu}^{a}\left(p_{+}, p_{-}\right)= & Z_{2} \gamma_{\mu}+\int_{q}^{\Lambda} K(p, q ; Q) \\
& \otimes S^{a}\left(q_{+}\right) \Gamma_{\mu}^{a}\left(q_{+}, q_{-}\right) S^{a}\left(q_{-}\right),
\end{aligned}
$$

where we ignore the possibility of flavor mixing in the kernel. There are twelve invariant amplitudes needed to represent the quark-photon vertex. The four longitudinal ones can be expressed directly in terms of the quark propagator amplitudes via the vector Ward-Takahashi identity [WTI]; the eight transverse amplitudes are defined by a decomposition of the form of Eq. (9) and are obtained from solutions of Eq. (10) as discussed in Ref. [13]. For timelike $Q^{2}$ near the position of a vector meson bound state with mass $m_{V}^{2}$, the transverse part of the quarkphoton vertex has the resonance pole behavior [13]

$$
\Gamma_{\mu}^{a}\left(p_{+}, p_{-}\right) \rightarrow \frac{f_{V} m_{V}}{Q^{2}+m_{V}^{2}} \Gamma_{\mu}^{a \bar{a} V}\left(p_{+}, p_{-}\right),
$$

where $f_{V}$ is the electroweak decay constant of the vector meson.

\section{B. Model Truncation}

We employ the model that has been developed recently for an efficient description of the masses and 
decay constants of the light pseudoscalar and vector mesons 10, 11]. This consists of the rainbow truncation of the DSE for the quark propagator and the ladder truncation of the BSE for the mesons. The required effective $\bar{q} q$ interaction has a phenomenological infrared behavior and reduces to the perturbative QCD running coupling in the ultraviolet 10, 11. In particular, the rainbow truncation of the quark DSE, Eq. (4), is

$$
Z_{1} g^{2} D_{\mu \nu}(k) \Gamma_{\nu}^{i}(q, p) \rightarrow \mathcal{G}\left(k^{2}\right) D_{\mu \nu}^{\text {free }}(k) \gamma_{\nu} \frac{\lambda^{i}}{2},
$$

where $D_{\mu \nu}^{\text {free }}(k=p-q)$ is the free gluon propagator in Landau gauge. The consistent ladder truncation of the BSE, Eq. (5), is

$$
K(p, q ; P) \rightarrow-\mathcal{G}\left(k^{2}\right) D_{\mu \nu}^{\text {free }}(k) \frac{\lambda^{i}}{2} \gamma_{\mu} \otimes \frac{\lambda^{i}}{2} \gamma_{\nu},
$$

where $k=p-q$. These two truncations are consistent in the sense that the combination produces vector and axialvector vertices satisfying the respective WTIs. In the axial case, this ensures that in the chiral limit the ground state pseudoscalar mesons are the massless Goldstone bosons associated with chiral symmetry breaking 10, 16. In the vector case, this ensures electromagnetic current conservation if the impulse approximation is used to describe the meson electromagnetic current or charge form factor 15]. Furthermore, this truncation was found to be particularly suitable for the flavor octet pseudoscalar and vector mesons since the next-order contributions in a quark-gluon skeleton graph expansion, have a significant amount of cancellation between repulsive and attractive corrections [17].

The model is completely specified once a form is chosen for the "effective coupling" $\mathcal{G}\left(k^{2}\right)$. We employ the Ansatz 11]

$$
\begin{aligned}
\frac{\mathcal{G}\left(k^{2}\right)}{k^{2}}= & \frac{4 \pi^{2} D k^{2}}{\omega^{6}} \mathrm{e}^{-k^{2} / \omega^{2}} \\
& +\frac{4 \pi^{2} \gamma_{m} \mathcal{F}\left(k^{2}\right)}{\frac{1}{2} \ln \left[\tau+\left(1+k^{2} / \Lambda_{\mathrm{QCD}}^{2}\right)^{2}\right]},
\end{aligned}
$$

with $\gamma_{m}=12 /\left(33-2 N_{f}\right)$ and $\mathcal{F}(s)=\left(1-\exp \frac{-s}{4 m_{t}^{2}}\right) / s$. The ultraviolet behavior is chosen to be that of the QCD running coupling $\alpha\left(k^{2}\right)$; the ladder-rainbow truncation then generates the correct perturbative QCD structure of the DSE-BSE system of equations. The first term implements the strong infrared enhancement in the region $0<k^{2}<1 \mathrm{GeV}^{2}$ phenomenologically required [18] to produce a realistic value for the chiral condensate. We use $m_{t}=0.5 \mathrm{GeV}, \tau=\mathrm{e}^{2}-1$, $N_{f}=4, \Lambda_{\mathrm{QCD}}=0.234 \mathrm{GeV}$, and a renormalization scale $\mu=19 \mathrm{GeV}$ which is well into the perturbative domain 10, 11]. The remaining parameters, $\omega=0.4 \mathrm{GeV}$ and $D=0.93 \mathrm{GeV}^{2}$ along with the quark masses, are fitted to give a good description of the chiral condensate, $m_{\pi / K}$ and $f_{\pi}$.

Within this model, the quark propagator reduces to the one-loop perturbative QCD propagator in the ultraviolet region. In the infrared region both the wave
TABLE I: Overview of the results of the model for the meson masses and decay constant, adapted from Refs. 110, 11.

\begin{tabular}{l|cc}
\hline \hline & $\begin{array}{r}\text { experiment [23] } \\
\text { (estimates) }\end{array}$ & $\begin{array}{r}\text { calculated } \\
\left({ }^{\dagger} \text { fitted) }\right.\end{array}$ \\
\hline$m_{\mu=1 \mathrm{GeV}}^{u=d}$ & $5-10 \mathrm{MeV}$ & $5.5 \mathrm{MeV}$ \\
$m_{\mu=1 \mathrm{GeV}}^{s}$ & $100-300 \mathrm{MeV}$ & $125 \mathrm{MeV}$ \\
\hline$-\langle\bar{q} q\rangle_{\mu}^{0}$ & $(0.236 \mathrm{GeV})^{3}$ & $\left(0.241^{\dagger}\right)^{3}$ \\
$m_{\pi}$ & $0.1385 \mathrm{GeV}$ & $0.138^{\dagger}$ \\
$f_{\pi}$ & $0.131 \mathrm{GeV}$ & $0.131^{\dagger}$ \\
$m_{K}$ & $0.496 \mathrm{GeV}$ & $0.497^{\dagger}$ \\
$f_{K}$ & $0.160 \mathrm{GeV}$ & 0.155 \\
\hline$m_{\rho}$ & $0.770 \mathrm{GeV}$ & 0.742 \\
$f_{\rho}$ & $0.216 \mathrm{GeV}$ & 0.207 \\
$m_{K^{\star}}$ & $0.892 \mathrm{GeV}$ & 0.936 \\
$f_{K^{\star}}$ & $0.225 \mathrm{GeV}$ & 0.241 \\
$m_{\phi}$ & $1.020 \mathrm{GeV}$ & 1.072 \\
$f_{\phi}$ & $0.236 \mathrm{GeV}$ & 0.259 \\
\hline \hline
\end{tabular}

function renormalization $Z\left(p^{2}\right)=1 / A\left(p^{2}\right)$ and the dynamical mass function $M\left(p^{2}\right)=B\left(p^{2}\right) / A\left(p^{2}\right)$ deviate significantly from the perturbative behavior, due to chiral symmetry breaking. Recent comparisons [19, 20] of results from this rainbow DSE model to lattice QCD simulations [21, 22 provide semiquantitative confirmation of the behavior generated by the present DSE model: a significant enhancement of $M\left(p^{2}\right)$ and a modest enhancement of $A\left(p^{2}\right)$ below $1 \mathrm{GeV}^{2}$.

The vector meson masses and electroweak decay constants produced by this model are in good agreement with experiments [11], as can be seen from Table If. Without any readjustment of the parameters, this model agrees remarkably well with the most recent Jlab data 24] for the pion charge form factor $F_{\pi}\left(Q^{2}\right)$. Also the kaon charge radii and electromagnetic form factors are well described $[15,25$. The strong decays of the vector mesons into a pair of pseudoscalar mesons are also well-described within this model [26, 27].

\section{THE $\pi \gamma \gamma$ TRANSITION}

The symmetrized invariant amplitude for the coupling of a pion with momentum $K=-\left(Q_{1}+Q_{2}\right)$ to a pair of photons with helicities $\lambda_{1}$ and $\lambda_{2}$ and momenta $Q_{1}$ and $Q_{2}$ has the form $\mathcal{M}^{\lambda_{1} \lambda_{2}}=\epsilon_{\mu}^{\lambda_{1}} \Lambda_{\mu \nu}^{P \gamma \gamma} \epsilon_{\nu}^{\lambda_{2}}$ where $\epsilon_{\mu}^{\lambda}$ is a photon polarization vector. With consideration of the two flavor-labeled components of neutral pion and the two orderings of photons, the vertex can be decomposed as

$$
\Lambda_{\mu \nu}^{\pi^{0} \gamma \gamma}=\frac{2}{\sqrt{2}}\left[\left(\hat{Q}^{u}\right)^{2} \Lambda_{\mu \nu}^{u}-\left(\hat{Q}^{d}\right)^{2} \Lambda_{\mu \nu}^{d}\right]
$$

where $\hat{Q}^{a}$ is the electric charge of the $a$-flavored quark. Here $\Lambda_{\mu \nu}^{a}$ is the vertex contribution from a given ordering of the photons and from flavor component $a \bar{a}$ of the $\pi^{0}$. The factor of $\sqrt{2}$ in the denominator comes from the fla- 
vor weights in the $\pi^{0}$ state, $(u \bar{u}-d \bar{d}) / \sqrt{2}$, and the factor of 2 in the numerator comes from summation over the two orderings of the photon vertices. Isospin symmetry gives $\Lambda_{\mu \nu}^{u}=\Lambda_{\mu \nu}^{d}$; thus we have $\Lambda_{\mu \nu}^{\pi^{0} \gamma \gamma}=\sqrt{2} \Lambda_{\mu \nu}^{u} / 3$. The impulse approximation result for the vertex $\Lambda_{\mu \nu}^{u}$ is

$$
\begin{aligned}
\Lambda_{\mu \nu}^{u}\left(Q_{1} ; Q_{2}\right)= & e^{2} N_{c} \int_{k}^{\Lambda} \operatorname{Tr}\left[S\left(q_{2}\right) \Gamma^{\pi}\left(q_{2}, q_{1}\right) S\left(q_{1}\right)\right. \\
& \left.\times i \Gamma_{\mu}\left(q_{1}, k\right) S(k) i \Gamma_{\nu}\left(k, q_{2}\right)\right]
\end{aligned}
$$

with $q_{1}=k+Q_{1}$ and $q_{2}=k-Q_{2}$. The result is independent of the choice of integration variable, and this provides a useful check on numerical methods.

The form factor that we associate with this process is defined from 28

$$
\begin{aligned}
& \Lambda_{\mu \nu}^{\pi^{0} \gamma \gamma}\left(Q_{1} ; Q_{2}\right)=\frac{\sqrt{2}}{3} \Lambda_{\mu \nu}^{u}\left(Q_{1}, Q_{2}\right)= \\
& \quad=\frac{2 i \alpha_{\mathrm{em}} g_{\pi \gamma \gamma}}{\pi \tilde{f}_{\pi}} \epsilon_{\mu \nu \rho \sigma} Q_{1 \rho} Q_{2 \sigma} F_{\pi \gamma \gamma}\left(Q_{1}^{2}, Q_{2}^{2}\right),
\end{aligned}
$$

where $\tilde{f}_{\pi}=f_{\pi} / \sqrt{2}=92 \mathrm{MeV}$ and the fine structure constant is $\alpha_{\mathrm{em}}=e^{2} / 4 \pi$. The Lorentz scalar $Q_{1} \cdot Q_{2}$ does not occur as a third argument of the form factor since the pion mass-shell constraint fixes it in terms of those shown. The form factor so defined has the normalization $F_{\pi \gamma \gamma}(0,0)=1$ so that $g_{\pi \gamma \gamma}$ is the coupling constant. For on-shell photons, $Q_{1}^{2}=Q_{2}^{2}=0$, this vertex describes the neutral pion decay and the associated width is

$$
\Gamma_{\pi^{0} \gamma \gamma}=\frac{g_{\pi \gamma \gamma}^{2} \alpha_{\mathrm{em}}^{2} m_{\pi}^{3}}{16 \pi^{3} \tilde{f}_{\pi}^{2}} .
$$

This decay is governed by the axial anomaly, which leads to $g_{\pi \gamma \gamma}^{0}=1 / 2$ in chiral limit. For example, the corresponding decay width produced by Eq. (18) is $\Gamma=$ $7.7 \mathrm{eV}$, while the experimental value is $\Gamma=7.8 \mathrm{eV}$.

A consistent ladder-rainbow truncation of the DSEBSE system of equations preserves the symmetry constraints that dictate this anomaly [29, 30]. Our approach indeed reproduces the correct neutral pion decay width. The difference in the coupling constant produced by the finite size of $m_{\pi}$ is less than $2 \%$, which is our estimate of our numerical accuracy. Since the decay width is very sensitive to $m_{\pi}$, we use the experimental $m_{\pi}$ to convert the experimental width to the coupling constant $g_{\pi \gamma \gamma}^{\text {expt }}=$ $0.501 \pm 0.018$; our theoretical result is $g_{\pi \gamma \gamma}^{\mathrm{th}}=0.502$.

\section{A. $\gamma^{*} \pi \gamma$ transition form factor}

For one on-shell photon, we can define a transition form factor $F_{\gamma^{*} \pi \gamma}\left(Q^{2}\right)=F_{\pi \gamma \gamma}\left(Q^{2}, 0\right)$ for the process $\gamma^{*} \pi \rightarrow \gamma$, which has been measured by the CLEO and CELLO collaborations [31, 32]. Our numerical results for this form factor are presented in Fig. 1, and agree very well with the available data. The corresponding interaction radius, defined by $r^{2}=-6 F^{\prime}(0)$ is $r_{\pi \gamma \gamma}^{2}=0.39 \mathrm{fm}^{2}$.
This is in much better agreement with the experimental estimate 31 of $r_{\pi \gamma \gamma}^{2}=0.42 \pm 0.04 \mathrm{fm}^{2}$ than an earlier theoretical result 28] within the present DSE framework that had a considerably greater reliance upon phenomenological elements.

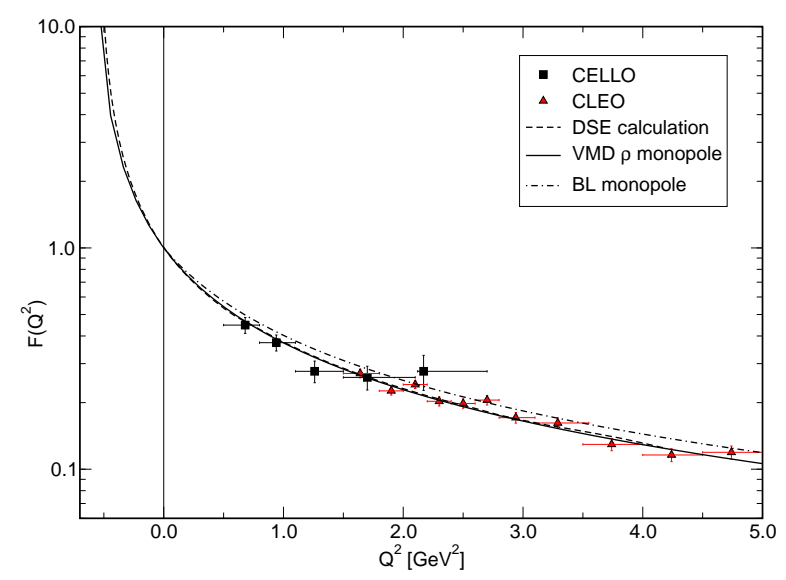

FIG. 1: Our results for the $\gamma^{*} \pi \rightarrow \gamma$ form factor, together with a simple VMD monopole with mass scale $m_{\rho}^{2}=$ $0.59 \mathrm{GeV}^{2}$, a monopole based on the Brodsky-Lepage asymptotic form $0.674 /\left(Q^{2}+0.674\right)$, and data from CLEO [32] and CELLO 31].

Our numerical results indicate that this form factor is an almost perfect monopole, and can be simulated very well by a VMD formula $F\left(Q^{2}\right)=m_{\rho}^{2} /\left(Q^{2}+m_{\rho}^{2}\right)$ with a $\rho$-meson mass scale $m_{\rho}^{2}=0.59 \mathrm{GeV}^{2}$, over the entire momentum range shown. With use of perturbative QCD and factorization on the lightfront, Brodsky and Lepage 33] showed that this form factor behaves like $Q^{2} F\left(Q^{2}\right) \rightarrow 8 \pi^{2}{\tilde{f_{\pi}}}^{2}=0.674 \mathrm{GeV}^{2}$ in the asymptotic spacelike region $\left(Q^{2}>0\right.$ here). Our results indicate a slightly lower asymptotic mass scale, see Fig. 11. We will discuss the asymptotic behavior further in Sec. III C below.

\section{B. Equal photon momenta}

Next, we consider the $\pi^{0} \gamma^{*} \gamma^{*}$ form factor, corresponding to an on-shell pion, and two off-shell photons that have equal spacelike virtualities $Q^{2}$. Although experimental data are not available for this equally virtual configuration, it is interesting from a theoretical point of view.

At low $Q^{2}$ this $\gamma^{*} \pi \rightarrow \gamma^{*}$ form factor is reasonably well represented by a dipole, in contrast to the $\gamma^{*} \pi \rightarrow \gamma$ case, which is an almost perfect monopole, see Fig. 2. This dipole behavior is consistent with VMD; in the symmetric case VMD leads to dipole behavior since both photons behave like $1 /\left(Q^{2}+m_{\rho}^{2}\right)$ near the $\rho$-pole. However, for spacelike momenta of about $Q^{2}>0.5 \mathrm{GeV}^{2}$, the influence of the vector meson resonance has weakened considerably 


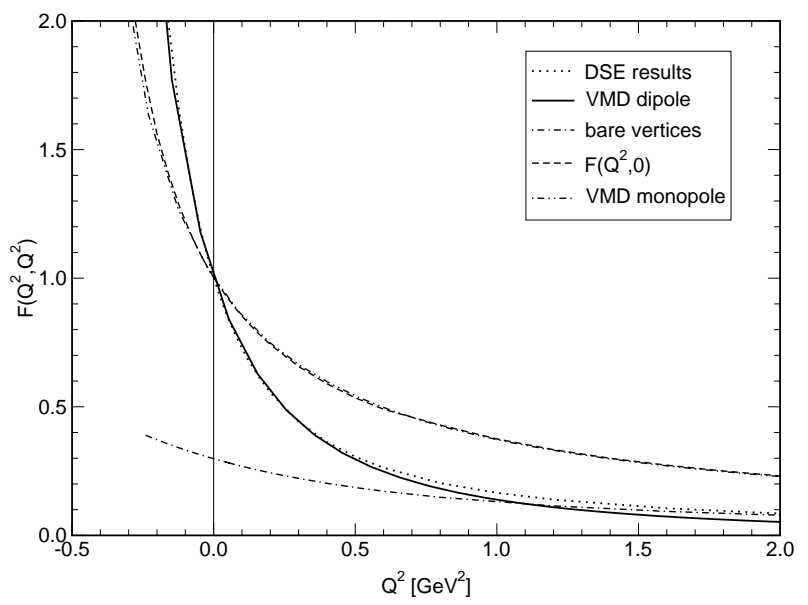

FIG. 2: Our DSE results for the symmetric $\gamma^{*} \pi \rightarrow \gamma^{*}$ form factor at low momentum together with a simple VMD dipole with mass scale $m_{\rho}^{2}=0.59 \mathrm{GeV}^{2}$. For comparison, the $\gamma^{*} \pi \rightarrow$ $\gamma$ form factor $F\left(Q^{2}, 0\right)$ and the corresponding VMD monopole from Fig. 11 are also shown.

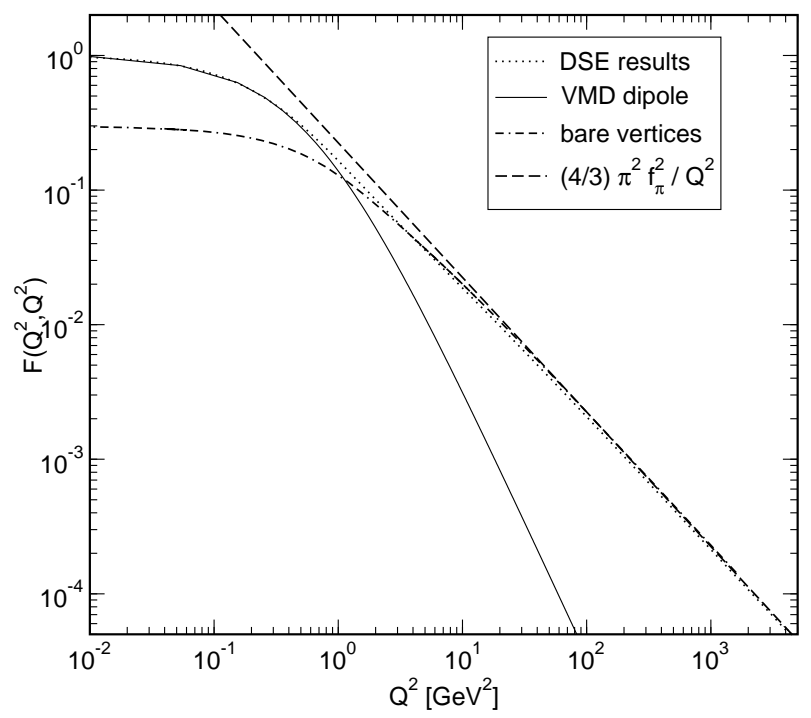

FIG. 3: Our DSE results for the symmetric $\gamma^{*} \pi \rightarrow \gamma^{*}$ form factor, compared to the derived asymptotic $1 / Q^{2}$ behavior. Note that $f_{\pi}=131 \mathrm{MeV}$. The naive VMD model suggests a dipole behavior which is correct only in the infrared.

and the behavior becomes increasingly a monopole form. This can be seen more clearly in Fig. 3 .

\section{Asymptotic behavior}

As pointed out recently [34], it is convenient to discuss the asymptotic behavior of this form factor with reference to the following form produced by the light-cone operator product expansion 33, 35

$$
F\left(Q_{1}^{2}, Q_{2}^{2}\right) \rightarrow 4 \pi^{2} \tilde{f}_{\pi}^{2}\left\{\frac{J(\omega)}{Q_{1}^{2}+Q_{2}^{2}}\right.
$$

$$
\left.+\mathcal{O}\left(\frac{\alpha_{s}}{\pi}, \frac{1}{\left(Q_{1}^{2}+Q_{2}^{2}\right)^{2}}\right)\right\} .
$$

Here $\omega$ is the photon asymmetry $\left(Q_{1}^{2}-Q_{2}^{2}\right) /\left(Q_{1}^{2}+Q_{2}^{2}\right)$. In lightfront QCD $J(\omega)$ is related to the leading twist pion distribution amplitude $\phi_{\pi}(x)$ by

$$
J(\omega)=\frac{4}{3} \int_{0}^{1} \frac{d x}{1-\omega^{2}(2 x-1)} \phi_{\pi}(x) .
$$

The normalization of $\phi_{\pi}(x)$ immediately gives $J(0)=\frac{4}{3}$ for the case of equal photon virtuality. Since a corresponding result does not exist for $\omega=1$, one expects more model dependence for that asymmetric case.

Within the present DSE-based approach, it is straightforward to analyze the asymptotic behavior for equal virtuality. From Eq. (16) with photon momenta $Q_{1}=$ $P-K / 2$ and $Q_{2}=-P-K / 2$, and pion momentum $K$, we have

$$
\begin{aligned}
\Lambda_{\mu \nu}^{u}\left(Q_{1} ; Q_{2}\right)= & -e^{2} N_{c} \int_{q}^{\Lambda} \operatorname{Tr}\left[S\left(q_{+}\right) \Gamma^{\pi}\left(q_{+}, q_{-}\right) S\left(q_{-}\right)\right. \\
& \left.\times \Gamma_{\mu}\left(q_{-}, \tilde{q}\right) S(\tilde{q}) \Gamma_{\nu}\left(\tilde{q}, q_{+}\right)\right] .
\end{aligned}
$$

where $q_{ \pm}=q \pm K / 2$, and $\tilde{q}=q+P$. The pion mass-shell condition coupled with $m_{\pi}$ being negligible compared to the other scales leads to the asymptotic domain being characterized by $Q_{1}^{2}=Q_{2}^{2} \simeq P^{2} \gg K^{2}$. In this perturbative domain, we have

$$
\begin{gathered}
\Gamma_{\mu}(q-K / 2, q+P) S(q+P) \Gamma_{\nu}(q+P, q+K / 2) \rightarrow \\
Z_{2}^{2} \gamma_{\mu} S^{0}(q+P) \gamma_{\nu}=-i Z_{2} \epsilon_{\mu \nu \alpha \beta} \gamma_{5} \gamma_{\alpha} P_{\beta} / P^{2} \\
+\cdots,
\end{gathered}
$$

where $Z_{2}$ is the renormalization constant appearing in Eq. (4) for the dressed quark propagator, and the terms not shown make only subleading contributions to the final result. Thus the asymptotic behavior of the impulse approximation vertex in Eq. (21) is

$$
\begin{aligned}
\Lambda_{\mu \nu}^{u}\left(Q_{1} ; Q_{2}\right)= & i e^{2} Z_{2} N_{c} \epsilon_{\mu \nu \alpha \beta} \frac{P_{\beta}}{P^{2}} \int_{q}^{\Lambda} \operatorname{Tr}\left[S\left(q_{+}\right)\right. \\
& \left.\Gamma^{\pi}\left(q_{+}, q_{-}\right) S\left(q_{-}\right) \gamma_{5} \gamma_{\alpha}\right] .
\end{aligned}
$$

Noting that the integral produces the pion decay constant $f_{\pi}$ via Eq. (8), we find from Eq. (17) the asymptotic result

$$
F\left(Q_{1}^{2}, Q_{2}^{2}\right) \rightarrow \frac{16 \pi^{2} \tilde{f}_{\pi}^{2}}{3\left(Q_{1}^{2}+Q_{2}^{2}\right)},
$$

or in other words, $J(0)=4 / 3$.

Our numerical calculation reproduces $J(0)=4 / 3$ within numerical accuracy as can be seen from the dashed curve in Fig. 3 where $Q_{1}^{2}=Q_{2}^{2}=Q^{2}$. In that Figure we also show that, in the asymptotic region, results employing the dressed quark-photon vertices obtained from solution of the ladder BSE, approach rapidly the results obtained with bare vertices. This confirms the key approximation used to obtain Eq. (23) for the analytic extraction of the asymptotic behavior within the DSE approach. 
TABLE II: The asymptotic behavior summarized in terms of the coefficient $J(1)$ for the $\gamma^{*} \pi \gamma$ form factor, and $J(0)$ for the symmetric $\gamma^{*} \pi \gamma^{*}$ form factor, as defined in Eq. (19).

\begin{tabular}{l|l}
\hline \hline $\mathrm{J}(1)$ lightcone pQCD 33, 35] & 2 \\
$\mathrm{~J}(1)$ Anikin et al. 34. & 1.8 \\
$\mathrm{~J}(1)$ earlier DSE analysis 36, 37, 38] & $4 / 3$ \\
$\mathrm{~J}(1)$ current DSE model numerical estimate & 1.7 \\
$\mathrm{~J}(1)$ experimental estimate & 1.6 \\
\hline $\mathrm{J}(0)$ Anikin et al. 34] & $4 / 3$ \\
$\mathrm{~J}(0)$ DSE analysis & $4 / 3$ \\
$\mathrm{~J}(0)$ current DSE model numerical result & 1.3 \\
\hline
\end{tabular}

Now we return to the experimentally accessible asymmetric case of one photon on-shell, and the other one virtual: $\omega=1$. Perturbative QCD on the lightfront, in combination with factorization, suggest $J(1)=2$ [33. However, a monopole fit to the experimental data, for $Q^{2}$ up to $10 \mathrm{GeV}^{2}$, produces $J(1)=1.6$ [34. This value agrees quite well with estimates based on a chiral quark model with a phenomenological interaction 34] and with a fit to numerical results of the DSE model of the present work. Table II provides a summary of these results.

The asymptotic behavior of the form factor for the asymmetric case $(\omega=1)$ was analyzed in earlier DSEbased works [36, 37, 38], through an argument that is the counterpart of what we have presented in Eqs. (23) and (24) for the symmetric case. A complicating aspect of the asymmetric case in Euclidean metric is that, because of the mass-shell constraint imposed by one photon being real, the two photon-quark vertices and the quark propagator linking them have momentum arguments containing imaginary parts that grow with the asymptotic scale, but are subdominant to the real parts. The earlier analyses made the reasonable assumption that the leading asymptotic behavior is produced by considering only the real arguments (and thus the known UV limit) of the propagator and vertices; the result obtained is $J(1)=4 / 3$. In the symmetric case, the scale of the imaginary parts is dictated only by $m_{\pi}$, and knowledge of the UV behavior of $\Gamma_{\mu} S \Gamma_{\nu}$ is needed only along the real spacelike momentum axis to produce the result given by Eq. (24). We consider this situation to be more reliable; it is confirmed by the comparison in Fig. 3 between results with and without dressing of the photon-quark vertices.

\section{THE $\rho \pi \gamma$ AND $\omega \pi \gamma$ TRANSITIONS}

An impulse approximation description of the $\rho^{0} \pi^{0} \gamma$, $\rho^{ \pm} \pi^{ \pm} \gamma$, and $\omega^{0} \pi^{0} \gamma$ transitions can be obtained simply by replacing one of the vector vertices in the generic $P V V$ triangle diagram, see Eq. (11), by the vector meson bound state BSA, and the other by the dressed quark-photon vertex. The only difference between these three radiative decays is in the flavor dependent factors.

For the radiative decay $\rho^{+} \rightarrow \pi^{+} \gamma$, the photon can ra- diate from either the $u$-quark or the $\bar{d}$-quark giving

$$
\Lambda_{\mu \nu}^{\rho^{+} \pi^{+} \gamma}=\hat{Q}_{u} \Lambda_{\mu \nu}^{u \bar{d}, u}+\hat{Q}_{\bar{d}} \Lambda_{\mu \nu}^{u \bar{d}, \bar{d}}
$$

where $\Lambda_{\mu \nu}^{u \bar{d}, q}$ is the vertex having the indicated quark flavor labeling and containing no charge or flavor weights associated with external bosons. With both the $\rho^{0}$ and $\pi^{0}$ given by $(u \bar{u}-d \bar{d}) / \sqrt{2}$, the radiative decay $\rho^{0} \rightarrow \pi^{0} \gamma$ can be expressed as

$$
\Lambda_{\mu \nu}^{\rho^{0} \pi^{0} \gamma}=\hat{Q}_{u} \Lambda_{\mu \nu}^{u \bar{u}, u}+\hat{Q}_{d} \Lambda_{\mu \nu}^{d \bar{d}, d}
$$

where the radiative contribution from both quark and antiquark of the same flavor have been combined. In the isospin symmetric limit, we have $\Lambda_{\mu \nu}^{u \bar{u}, u}=\Lambda_{\mu \nu}^{d \bar{d}, d}=$ $\Lambda_{\mu \nu}^{u \bar{d}, u}=-\Lambda_{\mu \nu}^{u \bar{d}, \bar{d}}$, and thus $\rho^{ \pm}$and $\rho^{0}$ have identical $\pi \gamma$ radiative decays at this level.

The radiative decay $\omega \rightarrow \pi^{0} \gamma$ is given by

$$
\Lambda_{\mu \nu}^{\omega \pi \gamma}=\hat{Q}_{u} \Lambda_{\mu \nu}^{u \bar{u}, u}-\hat{Q}_{d} \Lambda_{\mu \nu}^{d \bar{d}, d}
$$

where, compared to Eq. (26), the change in phase of the second term here comes from the phase of the $d \bar{d}$ component of the $\omega$. Again, with isospin symmetry, we have $\Lambda_{\mu \nu}^{\omega \pi \gamma}=\Lambda_{\mu \nu}^{u \bar{u}, u}$ and hence $\Lambda_{\mu \nu}^{\omega \pi \gamma}=3 \Lambda_{\mu \nu}^{\rho \pi \gamma}$.

In impulse approximation, the physical vertex is given by the quark loop integral

$$
\begin{aligned}
\Lambda_{\mu \nu}^{\rho \pi \gamma}(P ; Q)= & e \frac{N_{c}}{3} \int_{k}^{\Lambda} \operatorname{Tr}\left[S\left(q_{2}\right) \Gamma^{\pi}\left(q_{2}, q_{1}\right) S\left(q_{1}\right)\right. \\
& \left.\times \Gamma_{\mu}^{\rho}\left(q_{1}, k\right) S(k) i \Gamma_{\nu}^{\gamma}\left(k, q_{2}\right)\right]
\end{aligned}
$$

where $P$ is the $\rho$ momentum, the photon momentum is $Q$, and the pion momentum is $K=-(P+Q)$. We have used $q_{1}=k+P$ and $q_{2}=k-Q$. This is completely analogous to Eq. (16) for the $\pi \gamma \gamma$ vertex if the quarkphoton vertex $i \Gamma_{\mu}$ is replaced by the $\rho$ BSA. In fact, if that photon momenta is continued into the timelike region, use of Eq. (11) to extract the $\rho^{0}$ pole contribution to the $\pi \gamma \gamma$ vertex will identify Eq. (28) as the $\rho \pi \gamma$ vertex.

The $\rho \pi \gamma^{*}$ transition form factor is identified from the $\rho \pi \gamma$ vertex according to

$$
\Lambda_{\mu \nu}^{\rho \pi \gamma}(P ; Q)=\frac{e g_{\rho \pi \gamma}}{m_{\rho}} \epsilon_{\mu \nu \alpha \beta} P_{\alpha} Q_{\beta} F_{\rho \pi \gamma}\left(Q^{2}\right)
$$

at $P^{2}=-m_{\rho}^{2}$. At the photon point we have $F(0)=1$ and $g_{\rho \pi \gamma}$ is the conventional coupling constant associated with the radiative decay width

$$
\Gamma_{\rho \rightarrow \pi \gamma}=\frac{\alpha_{\mathrm{em}} g_{\rho \pi \gamma}^{2}}{24} m_{\rho}\left(1-\frac{m_{\pi}^{2}}{m_{\rho}^{2}}\right)^{3}
$$

The corresponding formula holds for the $\omega \rightarrow \pi \gamma$ decay with $g_{\omega \pi \gamma} / m_{\omega}=3 g_{\rho \pi \gamma} / m_{\rho}$.

As Eq. 29) shows, it is $g_{\rho \pi \gamma} / m_{\rho}$ that is the natural outcome of calculations of the vector radiative decays; therefore, it is this combination that we report in Table III. We also give the corresponding decay widths. 
TABLE III: Coupling constants and partial widths for radiative decays of $\rho$ and $\omega$, compared to experimental data 23.

\begin{tabular}{l|lc|lc}
\hline \hline & calc. $g / m$ & expt. $g / m$ & calc. $\Gamma$ & expt. $\Gamma$ \\
\hline$\rho^{0} \rightarrow \pi^{0} \gamma$ & $0.68 \mathrm{GeV}^{-1}$ & $0.9 \pm .2$ & $52 \mathrm{keV}$ & $102 \pm 25$ \\
$\rho^{ \pm} \rightarrow \pi^{ \pm} \gamma$ & 0.68 & $0.74 \pm .05$ & 52 & $68 \pm 7$ \\
$\omega^{0} \rightarrow \pi^{0} \gamma$ & 2.07 & $2.31 \pm .08$ & 479 & $717 \pm 43$ \\
\hline \hline
\end{tabular}

Except for the difference between the decay of the neutral and charged states of the $\rho$, which evidently is beyond the reach of the isospin symmetric impulse approximation, the agreement between theory and experiment is within $10 \%$. This is consistent with the other $\pi$ and $\rho$ observables obtained from the same model. Note that part of the difference between the experimental decay width and our calculated decay width comes from the phase space factor because our calculated $\rho$ and $\omega$ masses are about $5 \%$ too low.

\section{A. Transition form factor}

In Fig. A we show our DSE result for the $\omega \pi \gamma^{*}$ transition form factor as a function of the photon momentum $Q^{2}$. The $\rho \pi \gamma^{*}$ form factor is identical to this. Our result compares reasonably well with available experimental data for the $\omega \pi \gamma^{*}$ form factor in the timelike region [39]; we are not aware of data in the spacelike region. Also shown in Fig. A is a result from an earlier study [40] that implemented phenomenology at the level of the dressed quark propagator and the $\rho / \omega$ BSA. For reference we also display the simple VMD monopole having mass scale $m_{\omega}$.

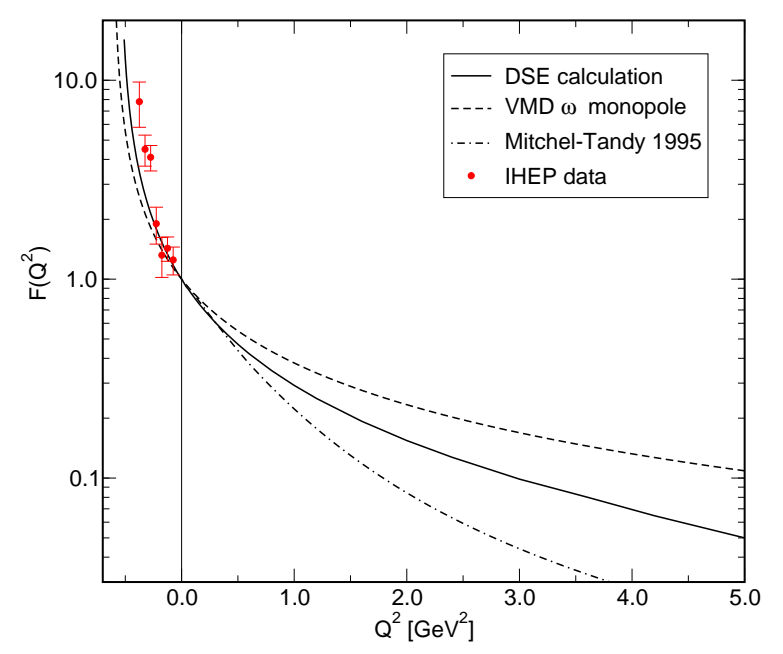

FIG. 4: Our DSE result (solid line) for the $\omega \pi \gamma^{*}$ (and $\rho \pi \gamma^{*}$ ) form factor, together with a simple VMD monopole with mass scale $m_{\omega}^{2}=0.61 \mathrm{GeV}^{2}$ (dashed line) and the more phenomenological result from Ref. 40]. The data in the timelike region are from Ref. [39].
Note that in the timelike region, the three different curves and the experimental data are all very close to each other: they are dominated by the resonance pole at the $\rho / \omega$ mass. However, the three curves differ significantly in the spacelike region where a monopole clearly does not represent the behavior obtained from modeling at quark-gluon level. Our results can be represented, in the manner of a Padé approximant, by the formula

$$
F_{\rho \pi \gamma}\left(Q^{2}\right)=\frac{1.0+Q^{2}}{1.0+3.04 Q^{2}+2.42 Q^{4}+0.36 Q^{6}},
$$

which is almost indistinguishable from our form factor on the domain $-0.5<Q^{2}<5 \mathrm{GeV}^{2}$. This suggests that the asymptotic behavior of this form factor is $1 / Q^{4}$. Although there are no experimental data available for the $\rho \pi \gamma^{*}$ process at spacelike photon momenta, indirect information about this form factor can be obtained from analyses of electron scattering from hadronic targets: e.g. it contributes as a meson exchange current tied to boson exchange models of the nuclear interaction. In this case the " $\rho \pi$ current" is virtual and the relationship to processes involving true quark-gluon bound states is not entirely clear.

Thus the $\rho \pi \gamma^{*}$ form factor plays a role in the interpretation of electron scattering data from light nuclei, because the isoscalar meson-exchange current contributes significantly to these processes. In particular our understanding of the deuteron EM structure functions for $Q^{2} \approx 2-6 \mathrm{GeV}^{2}$ requires knowledge of this form factor [41]. An initial exploratory study [40] of the $\rho \pi \gamma^{*}$ vertex within the present framework, but employing phenomenology to a much greater extent, produced a very soft result for the form factor and this is shown in Fig. 1 as the Mitchell-Tandy curve. It was found 41] that the resulting meson exchange current contribution provided a very good description of the elastic deuteron electromagnetic form factors $A\left(Q^{2}\right)$ and $B\left(Q^{2}\right)$ in the range $2-6 \mathrm{GeV}^{2}$ where such effects are important.

The model under consideration in the present work, based on the DSEs of QCD, has no free parameter other than the two set by $f_{\pi}$ and $\langle q \bar{q}\rangle$ as described earlier; the amount of phenomenology is significantly less than the earlier Mitchell-Tandy result [40]. The present work produces a form factor that is much softer than what is inferred from VMD but obviously harder than the Mitchell-Tandy result [40], as can be seen in Fig. 1 . We expect the present impulse approximation results for the form factor to be as reliable as the pion and kaon charge form factors from this model 15$]$. The present form factor is harder than what is required to fit electron scattering from the deuteron if the standard analysis is employed 42. What is required for progress is some estimation of the likely effect from the virtuality of the $\pi$ and $\rho \bar{q} q$ correlations relevant to the meson exchange current mechanism. An investigation of a related aspect of this issue is to be found below. 


\section{B. Virtual vector $\bar{q} q$ correlations and VMD}

In Sec. III A we have seen that the $\gamma^{*} \rightarrow \pi \gamma$ form factor is an almost perfect monopole with $m_{\rho}$ as the mass scale, see Fig. 11. This suggests that the vector meson resonance pole in the dressed $\bar{q} \gamma^{*} q$ vertex dominates to the extent that the following VMD mechanism is very effective: there is a $\gamma-\rho$ transition, a $\rho$ propagation, then a $\rho-\pi-\gamma$ transition. Implicit in this three-step mechanism, are the assumptions: that even for spacelike momentum of the $\gamma^{*}$, the first and third steps are described by coupling constants defined at the $\rho$ mass-shell, and the momentum dependence is carried totally by the propagator of a point particle having the $\rho$ mass. However, at the quark-gluon level, one cannot define a $\rho$-meson bound state at spacelike momentum; only a vector $\bar{q} q$ correlation with the same quantum numbers of the $\rho$ can be discussed. Hence the issue is the domain of applicability and the accuracy of those assumptions. We wish to explore this for a variety of PVV processes; we will begin with the connection between the $\gamma^{*} \pi \gamma$ process and the $\rho \pi \gamma$ process.

We begin with Eq. (16) for the quark loop integral which produces the vertex $\Lambda_{\mu \nu}^{\pi^{0} \gamma \gamma}$ via Eq. (17), use the notation change $Q_{1} \rightarrow P$ and $Q_{2} \rightarrow Q$, and consider the domain $P^{2} \approx-m_{\rho}^{2}$. The quark-photon vertex $\Gamma_{\mu}\left(q_{1}, k\right)$ in Eq. (16) then behaves like

$$
\Gamma_{\mu}\left(q_{1}, k\right) \sim \frac{f_{\rho} m_{\rho}}{P^{2}+m_{\rho}^{2}} \Gamma_{\mu}^{\rho}\left(q_{1}, k\right) .
$$

Use of only this resonance contribution gives

$$
\begin{aligned}
& \Lambda_{\mu \nu}^{\pi^{0} \gamma \gamma}(P ; Q) \sim \frac{f_{\rho} m_{\rho}}{P^{2}+m_{\rho}^{2}} \frac{\sqrt{2} e^{2} N_{c}}{3} \\
& \times \int_{k}^{\Lambda} \operatorname{Tr}\left[S\left(q_{2}\right) \Gamma^{\pi}\left(q_{2}, q_{1}\right) S\left(q_{1}\right) i \Gamma_{\mu}^{\rho}\left(q_{1}, k\right) S(k) i \Gamma_{\nu}\left(k, q_{2}\right)\right] \\
& =\frac{i e \sqrt{2} f_{\rho} m_{\rho}}{P^{2}+m_{\rho}^{2}} \Lambda_{\mu \nu}^{\rho \pi \gamma}(P ; Q)
\end{aligned}
$$

where the last equality follows from identifying the quark loop integral for the $\rho \pi \gamma$ vertex via Eq. (28). We substitute the general forms of both vertices from Eqs. (17) and (29) and cancel common factors. This yields

$$
\frac{g_{\pi \gamma \gamma}}{4 \pi^{2} \tilde{f}_{\pi}} F_{\gamma^{*} \pi \gamma^{*}}\left(P^{2}, Q^{2}\right) \sim \frac{f_{\rho} g_{\rho \pi \gamma}}{\sqrt{2}\left(P^{2}+m_{\rho}^{2}\right)} F_{\rho \pi \gamma^{*}}\left(Q^{2}\right) \text {. }
$$

When the non-resonant photon is real, Fig. 11 shows that $F_{\gamma^{*} \pi \gamma}\left(P^{2}, Q^{2}=0\right)$ is very accurately described by this monopole shape even for spacelike momentum as large as $P^{2}=5 \mathrm{GeV}^{2} \simeq 10 m_{\rho}^{2}$. At $P^{2}=0$ Eq. (34) accurately accounts for the transition strength $g_{\rho \pi \gamma}$ in terms of $g_{\pi \gamma \gamma}$ and the other more fundamental quantities. The relation

$$
\frac{g_{\pi \gamma \gamma}}{4 \pi^{2} \tilde{f}_{\pi}} \sim \frac{f_{\rho} g_{\rho \pi \gamma}}{\sqrt{2} m_{\rho}^{2}}
$$

is borne out to the extent that substitution of the separately calculated values of these quantities gives 0.138 for the LHS and 0.134 for the RHS.

In the $\gamma^{*} \rightarrow \pi \gamma$ process, we can view the $\pi \gamma$ transition current as coupling to a vector $\bar{q} q$ correlation initiated by the $\gamma^{*}$. In the process $\gamma^{*} N \rightarrow \pi N$ away from the $s$-channel resonance region, one often considers soft $t$-channel mechanisms modeled by meson exchange. In the case of a vector $t$-channel mechanism, the nucleon current is required to provide a spacelike vector $\bar{q} q$ correlation to the $\gamma \pi$ transition current. If we model the vector $\bar{q} q$ vertex provided by the nucleon current as $\bar{q}\left(p_{+}\right) \gamma_{\mu} g_{V N N}\left(P^{2}\right) q\left(p_{-}\right)$, where $g_{V N N}\left(P^{2}\right)$ is the phenomenological coupling constant and form factor corresponding to the $t$-channel momentum $P$, then we have a point $\bar{q} q$ correlation that can interact and propagate to the $\gamma \pi$ transition current. The object with momentum $P=p_{+}-p_{-}$that describes this satisfies the inhomogeneous BSE

$$
\begin{aligned}
\Gamma_{\mu}^{V N N}\left(p_{+}, p_{-}\right)= & Z_{2} \gamma_{\mu} g_{V N N}\left(P^{2}\right)+\int_{q}^{\Lambda} K(p, q ; P) \\
& \otimes S\left(q_{+}\right) \Gamma_{\mu}^{V N N}\left(q_{+}, q_{-}\right) S\left(q_{-}\right) .(36)
\end{aligned}
$$

This is just $g_{V N N}\left(P^{2}\right)$ times the dressed vertex seeded by $\gamma_{\mu}$; i.e., $g_{V N N}\left(P^{2}\right)$ times the photon vertex $\Gamma_{\mu}$. Since $\Gamma_{\mu}$ has the vector meson pole, see Eq. (32), the meson exchange mechanism, including the "meson" propagation, is embedded in this vertex.

A more exact treatment would be to calculate a distributed vector $\bar{q} q$ vertex $\bar{q}\left(p_{+}\right) \mathcal{V}_{\mu}\left(p_{+}, p_{-}\right) q\left(p_{-}\right)$from a quark-gluon model of the nucleon. In the absence of a reliable calculation of such a distributed vertex, we here explore the consequences of the above modeling of the nucleon current as a pointlike current $\bar{q}\left(p_{+}\right) \gamma_{\mu} g_{V N N}\left(P^{2}\right) q\left(p_{-}\right)$. For simplicity, we omit the phenomenological nucleon coupling strength $g_{V N N}\left(P^{2}\right)$ in the calculations below, which can be easily appended for subsequent applications such as $\gamma^{*} N \rightarrow \pi N$ processes. Thus, for the $V^{*} \pi \gamma^{*}$ vertex we are led to the impulse approximation

$$
\begin{aligned}
\Lambda_{\mu \nu}^{V \pi \gamma}(P ; Q)= & e \frac{N_{c}}{3} \int_{k}^{\Lambda} \operatorname{Tr}\left[S\left(q_{2}\right) \Gamma^{\pi}\left(q_{2}, q_{1}\right) S\left(q_{1}\right)\right. \\
& \left.\times \Gamma_{\mu}^{V}\left(q_{1}, k\right) S(k) i \Gamma_{\nu}^{\gamma}\left(k, q_{2}\right)\right]
\end{aligned}
$$

where $Q$ is the photon momentum, the vector current momentum is $P$, and the pion momentum is $K=-(P+Q)$. We have used $q_{1}=k+P$ and $q_{2}=k-Q$ in complete analogy to Eq. 28) for the $\rho \pi \gamma$ vertex but with $\Gamma_{\mu}^{V}$ replacing the $\rho$ BSA. In analogy with Eq. (29), the general form may be expressed as

$$
\Lambda_{\mu \nu}^{V \pi \gamma}(P ; Q)=\frac{e g_{\rho \pi \gamma}}{m_{\rho}} \epsilon_{\mu \nu \alpha \beta} P_{\alpha} Q_{\beta} A_{V^{*} \pi \gamma^{*}}\left(P^{2}, Q^{2}\right),
$$

thereby defining an amplitude $A_{V^{*} \pi \gamma^{*}}$. Due to Eq. (32), this amplitude has the physical vector meson resonance 
pole at the mass-shell $P^{2}=-m_{\rho}^{2}$. We thus may write for any momentum

$$
A_{V^{*} \pi \gamma^{*}}\left(P^{2}, Q^{2}\right)=\frac{f_{\rho} m_{\rho}}{P^{2}+m_{\rho}^{2}} G_{\rho^{*} \pi \gamma^{*}}\left(P^{2}, Q^{2}\right),
$$

and this serves to define a generalized form factor $G_{\rho^{*} \pi \gamma^{*}}$ for the "process" $\gamma^{*} \rho^{*} \rightarrow \pi$. At the $\rho$ mass-shell, this form factor $G_{\rho^{*} \pi \gamma^{*}}$ reduces to the previously defined form factor $F_{\rho \pi \gamma^{*}}\left(Q^{2}\right)$. Note that this form factor is not unique in the sense that we have chosen one particular description of the vector $\bar{q} q$ correlation. A different choice for the inhomogeneous term in Eq. (36) would lead to a different amplitude $A_{V^{*} \pi \gamma^{*}}$ and thus to a different form factor $G_{\rho^{*} \pi \gamma^{*}}$; only for on-shell $\rho$ and $\pi$ mesons can one uniquely define a form factor $F_{\rho \pi \gamma^{*}}$.

Our choice for the inhomogeneous term leads to a momentum dependence of the amplitude $A_{V^{*} \pi \gamma^{*}}$ that is the same as that of the $\gamma^{*} \pi \gamma^{*}$ form factor, apart from the overall scale. Hence the earlier observations associated with Fig. 1 indicate that, for $Q^{2} \approx 0$ the monopole shape in $P^{2}$ with mass scale $m_{\rho}$ dominates. Thus the form factor $G_{\rho^{*} \pi \gamma^{*}}\left(P^{2}, Q^{2} \simeq 0\right)$ in Eq. (39) has very little $P^{2}$ dependence and the meson exchange picture is thus very effective there. In other words, the strict VMD approximation to Eq. (39)

$$
A_{V^{*} \pi \gamma^{*}}\left(P^{2}, Q^{2}\right) \approx \frac{f_{\rho} m_{\rho}}{P^{2}+m_{\rho}^{2}} F_{\rho \pi \gamma^{*}}\left(Q^{2}\right),
$$

is valid for $Q^{2} \simeq 0$ and for the complete range of spacelike $P^{2}$ investigated here $\left(<5 \mathrm{GeV}^{2}\right)$.

The question arises: what is the more general domain of $Q^{2}$ and $P^{2}$ for the applicability of the meson exchange picture? Certainly when $Q^{2}$ and $P^{2}$ are both large, we can conclude from Fig. 3 that the amplitude $A_{V^{*} \pi \gamma^{*}}\left(P^{2}, Q^{2}\right)$ with $Q^{2}=P^{2}$ falls off like $1 / P^{2}$, whereas a meson dominance model would predict $1 / P^{4}$. Thus, the form factor $G_{\rho^{*} \pi \gamma^{*}}\left(P^{2}, P^{2}\right)$ becomes a constant for large $P^{2}$, in contrast to $F_{\rho \pi \gamma^{*}}\left(P^{2}\right)$ which seems to fall off as $1 / P^{4}$, as can be seen from Fig. 1 and our fit, Eq. (31).

In the top panel of Fig. 5 we display as discrete data points our results for the $P^{2}$-dependence of the amplitude $A_{V^{*} \pi \gamma^{*}}\left(P^{2}, Q^{2}\right)$ for several values of the photon momentum $Q^{2}$, and compare it with the naive VMD formula, Eq. (40). For photon momenta $Q^{2}$ near the $\rho$-pole, the behavior of the amplitude $A$ as function of $P^{2}$ is softer than one would expect from VMD. This is no surprise, since we have already seen in the previous section that the on-shell $F_{\rho \pi \gamma^{*}}$ is softer than VMD. For $Q^{2} \approx 0$, the VMD prediction works quite well. For increasing spacelike $Q^{2}$ the amplitude $A_{V^{*} \pi \gamma^{*}}$ is progressively harder in $P^{2}$ (i.e. falls off slower) compared to the VMD approximation. This means that the $1 /\left(P^{2}+m_{\rho}^{2}\right)$ behavior of the $\rho$-meson propagator increasingly over-estimates the falloff with momentum of the virtual vector object; the meson exchange picture is showing its limitations. As a consequence, the momentum dependence of the generalized form factor $G_{\rho^{*} \pi \gamma^{*}}\left(P^{2}, Q^{2}\right)$ becomes more evident;
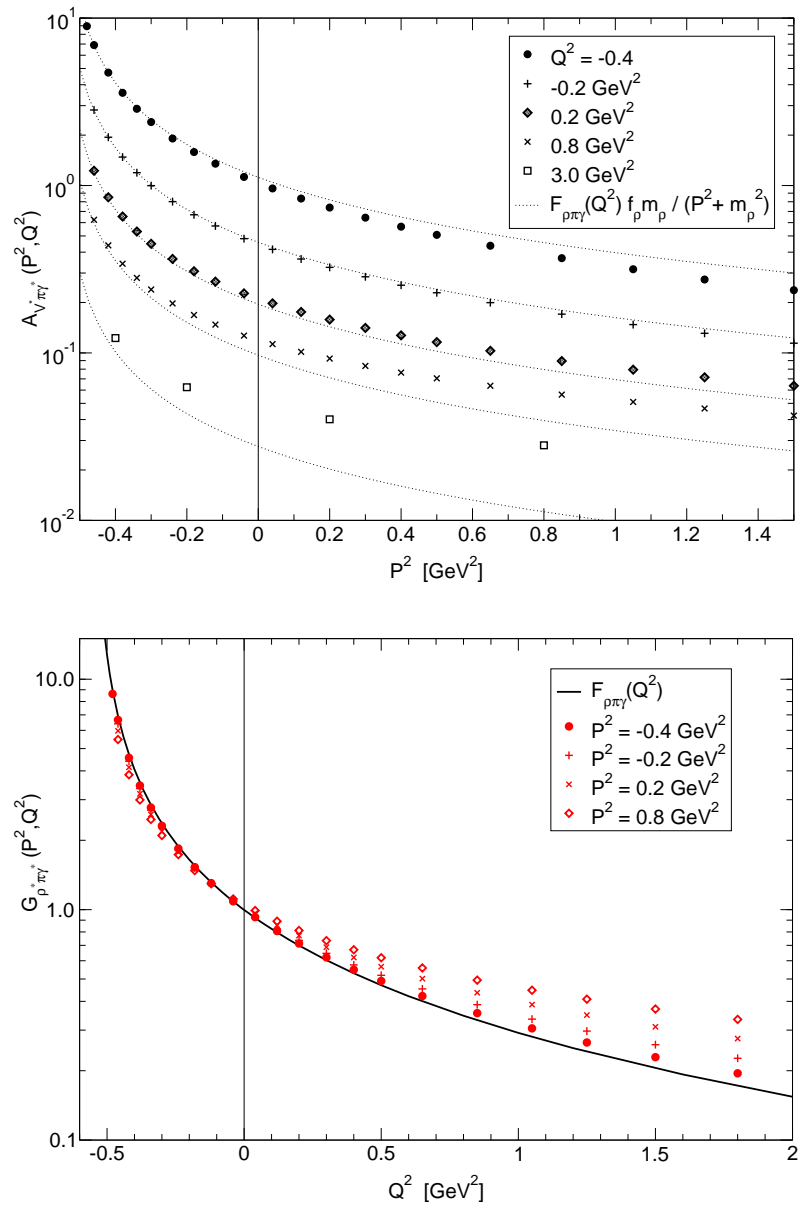

FIG. 5: Top: The virtual vector momentum $P^{2}$-dependence of the amplitude $A_{V^{*} \pi \gamma^{*}}$ from the DSE calculation for several values of the photon momentum $Q^{2}$ is shown by the discrete points. The meson exchange approximation is shown by the curves. Bottom: The $Q^{2}$-dependence of the generalized form factor $G_{\rho^{*} \pi \gamma^{*}}\left(P^{2}, Q^{2}\right)$ for several values of the virtual vector momentum $P^{2}$ is shown by the discrete points. The curve is the on-shell $F_{\rho \pi \gamma^{*}}\left(Q^{2}\right)$.

compared to the on-shell form factor $F_{\rho \pi \gamma^{*}}\left(Q^{2}\right)$ it becomes progressively harder in $Q^{2}$ for increasing (spacelike) $P^{2}$. This can be seen more clearly in the bottom panel of Fig. 同.

If $Q^{2}$ and $P^{2}$ are both more spacelike than about $1 \mathrm{GeV}^{2}$, the point meson propagator in the VMD or meson exchange approximation represented by Eq. (40) can overestimate the falloff by $50 \%$ or more. We anticipate this inadequacy of the point meson propagator to apply generally to meson exchange models. No standard phenomenological form factors for the coupling of virtual "mesons" to hadronic currents ( $\operatorname{such}$ as $g_{V N N}\left(P^{2}\right)$ ) can compensate for the propagator falling off too fast. In the present specific case, we have a spacelike $\bar{q} \gamma_{\mu} q$ correlation, not a "virtual bound state meson". This $\bar{q} \gamma_{\mu} q$ correlation falls off slower than a point meson propagator; in the UV region it does not vanish, but goes to a bare vertex, $\gamma_{\mu}$. Our modeling of the $\bar{q} \gamma_{\mu} q$ correlation 
by a ladder BSE with a point inhomogeneous term is in agreement with perturbative QCD, whereas the notion of a highly virtual $\rho$-meson "bound state" does not make any sense in the perturbative region.

\section{Virtual pseudoscalar $\bar{q} q$ correlations}

As an extension of these observations, we also calculate the corresponding amplitude associated with the $\rho P^{*} \gamma^{*}$ vertex, where $P$ stands for the pointlike pseudoscalar current $\bar{q} \gamma_{5} q$. The interaction and propagation of this correlation generates the pion pole. The quantity thus made available to us is relevant to the $\gamma^{*} N \rightarrow \rho N$ process via $t$-channel exchange of a pseudoscalar $\bar{q} q$ correlation. Of course, in a more realistic calculation, one should use a distributed vertex $\bar{q}\left(p_{+}\right) \mathcal{P}\left(p_{+}, p_{-}\right) q\left(p_{-}\right)$from a quarkgluon model of the nucleon, rather than a pointlike pseudoscalar vertex. Corresponding to Eq. (36) for the vector case, our approximation for the pseudoscalar $\bar{q} q$ correlation (with the phenomenological $g_{P N N}\left(K^{2}\right)$ removed) is the inhomogeneous BSE for the vertex

$$
\begin{aligned}
\Gamma_{5}\left(p_{+}, p_{-}\right)= & Z_{4} \gamma_{5}+\int_{q}^{\Lambda} K(p, q ; Q) \\
& \otimes S\left(q_{+}\right) \Gamma_{5}\left(q_{+}, q_{-}\right) S\left(q_{-}\right),
\end{aligned}
$$

where the total momentum is $K=p_{+}-p_{-}$. Note that this dressed vertex is renormalization-point dependent; however, the combination $m_{q}(\mu) \Gamma_{5}$, where $m_{q}(\mu)$ is the current quark mass, is renormalization-point independent. Near the pion pole, this dressed vertex $\Gamma_{5}$ behaves like

$$
\Gamma_{5}\left(p_{+}, p_{-}\right) \sim \frac{r_{P}(\mu)}{K^{2}+m_{\pi}^{2}} \Gamma^{\pi}\left(p_{+}, p_{-}\right)
$$

where $r_{P}(\mu)$ is the renormalization-point-dependent residue in the pseudoscalar channel and is given by 16

$$
r_{P}(\mu)=Z_{4} N_{c} \int_{q}^{\Lambda} \operatorname{Tr}\left[\gamma_{5} S\left(q_{+}\right) \Gamma^{\pi}\left(q_{+}, q_{-}\right) S\left(q_{-}\right)\right]
$$

The axial-vector WTI dictates that this pseudoscalar residue is related to the pion mass and decay constant through 16.

$$
2 m_{q}(\mu) r_{P}(\mu)=f_{\pi} m_{\pi}^{2}
$$

where the renormalization-point dependence of the current quark mass and that of the residue is such that the combination is renormalization-point independent.

In a way that is completely parallel to Eq. (37), we model the $\rho P^{*} \gamma^{*}$ vertex through the impulse approximation

$$
\begin{aligned}
\Lambda_{\mu \nu}^{\rho P \gamma}(P ; Q)= & e \frac{N_{c}}{3} \int_{q}^{\Lambda} \operatorname{Tr}\left[S\left(q_{2}\right) \Gamma_{5}\left(q_{2}, q_{1}\right) S\left(q_{1}\right)\right. \\
& \left.\times \Gamma_{\mu}^{\rho}\left(q_{1}, k\right) S(k) i \Gamma_{\nu}^{\gamma}\left(k, q_{2}\right)\right],
\end{aligned}
$$

and note that this is renormalization-point dependent. We can define a renormalization-point dependent amplitude $A_{\rho P^{*} \gamma^{*}}\left(K^{2}, Q^{2}\right)$ via

$$
\begin{aligned}
& \Lambda_{\mu \nu}^{\rho P \gamma}(P ; Q)= \\
& \quad e \frac{g_{\rho \pi \gamma}}{m_{\rho}} \epsilon_{\mu \nu \alpha \beta} P_{\alpha} Q_{\beta} A_{\rho P^{*} \gamma^{*}}\left(K^{2}, Q^{2}\right),
\end{aligned}
$$

where $K=-(P+Q)$ is the pseudoscalar momentum and $Q$ is the photon momentum, both incoming to the diagram. This amplitude $A_{\rho P^{*} \gamma^{*}}$ has the physical pion resonance pole at the mass-shell $K^{2}=-m_{\pi}^{2}$. We thus may write for any momentum

$$
A_{\rho P^{*} \gamma^{*}}\left(K^{2}, Q^{2}\right)=\frac{r_{P}(\mu)}{K^{2}+m_{\pi}^{2}} G_{\rho \pi^{*} \gamma^{*}}\left(K^{2}, Q^{2}\right),
$$

and this serves to define a generalized form factor $G_{\rho \pi^{*}} \gamma^{*}$ for the "process" $\gamma^{*} P^{*} \rightarrow \rho$. This dimensionless form factor is renormalization-point independent, and reduces to the on-shell form factor $F_{\rho \pi \gamma^{*}}\left(Q^{2}\right)$ at the pion massshell $K^{2}=-m_{\pi}^{2}$. With $G_{\rho \pi^{*} \gamma^{*}}$ replaced by its value at the pion mass-shell, Eq. (47) yields the meson exchange approximation

$$
A_{\rho P^{*} \gamma^{*}}\left(K^{2}, Q^{2}\right) \approx \frac{r_{P}(\mu)}{K^{2}+m_{\pi}^{2}} F_{\rho \pi \gamma^{*}}\left(Q^{2}\right) .
$$

The directly calculated amplitude $A_{\rho P^{*} \gamma^{*}}$ is shown in the upper panel of Fig. 6 by the discrete points as a function of $K^{2}$ for several values of $Q^{2}$. The continuous curves illustrate the meson exchange approximation, Eq. (48). The degree of agreement indicates the domain where the pseudoscalar meson exchange picture is effective. The pion pole dominates the behavior at low momentum of the pseudoscalar correlation. For on-shell photons, the meson exchange approximation is quite accurate for spacelike up to about $K^{2}=1 \mathrm{GeV}^{2} \approx 50 m_{\pi}^{2}$. However with increasing $Q^{2}$, one observes that the pion pole alone falls off with $K^{2}$ faster than what is required to describe $A_{\rho P^{*} \gamma^{*}}\left(K^{2}, Q^{2}\right)$. Similar to the vector case, for spacelike photon momenta, the falloff of the amplitude $A$ with $K^{2}$ is slower than that of a pseudoscalar meson dominance model; the meson exchange picture thus has a very limited domain of applicability.

In the bottom panel of Fig. 6 we display the generalized form factor $G_{\rho \pi^{*} \gamma^{*}}\left(K^{2}, Q^{2}\right)$ as a function of the photon momentum $Q^{2}$ for several values of the virtual pseudoscalar $K^{2}$. The physical form factor at the pseudoscalar meson mass-shell, $F_{\gamma^{*} \rho \pi}\left(Q^{2}\right)$, is shown by the continuous curve. The rise at timelike photon momentum is due to the vector pole in the photon-quark vertex. This figure illustrates that the form factor as a function of $Q^{2}$ becomes harder as the momentum $K^{2}$ of the spacelike pseudoscalar correlation is increased. For spacelike $K^{2}<0.1 \mathrm{GeV}^{2} \approx 5 m_{\pi}^{2}$ the meson exchange approximation is quite good up to $Q^{2} \approx 3 \mathrm{GeV}^{2}$, but with the larger virtuality $K^{2}>0.8 \simeq 40 m_{\pi}^{2}$ the error in the meson dominance assumption has grown to almost a factor 

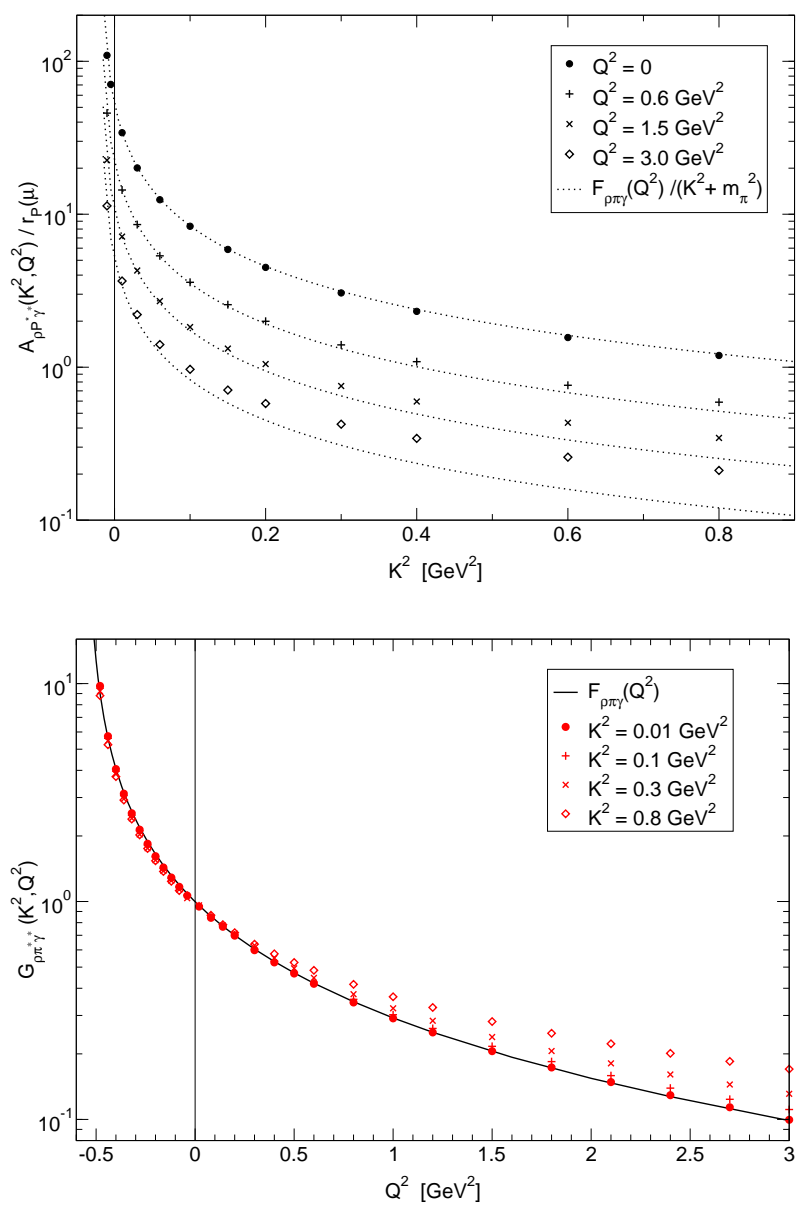

FIG. 6: Top: The dependence of the amplitude $A_{\rho P^{*} \gamma^{*}}$ on the pseudoscalar momentum $K^{2}$ for several values of the photon momentum $Q^{2}$ is given by the discrete points from the DSE calculation. The meson exchange approximation is given by the curves. Bottom: The DSE results for the $Q^{2}$ dependence of the generalized form factor $G_{\rho \pi^{*} \gamma^{*}}\left(K^{2}, Q^{2}\right)$ for several values of the pseudoscalar momentum $K^{2}$ are the discrete points. The on-shell $F_{\rho \pi \gamma^{*}}\left(Q^{2}\right)$ is given by the curve.

of 2 at $Q^{2} \approx 3 \mathrm{GeV}^{2}$. No standard phenomenological form factor for the meson-nucleon coupling, $g_{P N N}\left(K^{2}\right)$, can compensate. This observation is evidently due to the fact that the $\bar{q} \gamma_{5} q$ correlation does not continue to fall off with increasing spacelike total momentum, but goes to a constant.

\section{SUMMARY}

We have studied selected meson transition processes and associated form factors within a model of QCD based on the Dyson-Schwinger equations truncated to ladderrainbow level. The infrared structure of the ladderrainbow kernel is described by two parameters; the ultraviolet behavior is fixed by the one-loop renormalization group behavior of QCD. Within the $u$ and $d$ quark sector we have obtained the coupling constants for the radiative decays: $\rho \rightarrow \pi \gamma, \omega \rightarrow \pi \gamma$, and $\pi^{0} \rightarrow \gamma \gamma$. We have studied the form factors for the associated transitions: $\gamma^{*} \pi^{0} \rightarrow \gamma, \gamma^{*} \pi^{0} \rightarrow \gamma^{*}, \gamma^{*} \pi \rightarrow \rho$ and $\gamma^{*} \rho \rightarrow \pi$. The latter two processes are of interest as contributors to meson electroproduction from hadronic targets away from the $s$-channel resonance region.

We have exploited the fact that since a quark-gluon model can dynamically produce the vector meson pole in the dressed photon-quark vertex, the validity and effectiveness of using a meson exchange picture in nearby momentum domains can be tested. We find that for the transition $\gamma^{*} \pi^{0} \rightarrow \gamma$, the vector meson resonance pole term extrapolated to the photon point produces an estimate of the $\rho$ radiative decay coupling constant $g_{\rho \pi \gamma}$ in terms of the $\pi$ decay coupling constant $g_{\pi \gamma \gamma}$ that is accurate to within a few percent. However at the photon point and more generally for spacelike momentum, there is no vector meson bound state; the object that occurs in such dynamics is a vector $\bar{q} q$ correlation described by the dressed $\bar{q} \gamma_{\mu} q$ vertex. This particular meson- $\bar{q} q$ duality cannot survive when the momentum of the vector object becomes sufficiently large and spacelike because the dressed $\bar{q} \gamma_{\mu} q$ vertex eventually becomes bare or perturbative, it does not fall off with large spacelike momentum; that is, it cannot provide a fall-off like a point meson propagator. Nevertheless, for a large range of spacelike momentum, we find the shape of the form factor for the transition $\gamma^{*} \pi^{0} \rightarrow \gamma$ to be quite accurately described by a monopole with mass scale $m_{\rho}$; that is also consistent with analyses of the asymptotic behavior. This is an example of the empirical effectiveness of the simple VMD assumption being much greater than its faithfulness to the underlying dynamics or physical picture.

We have examined this issue further by considering form factors for transition processes $\gamma^{*} P \rightarrow \rho$ and $\gamma^{*} V \rightarrow \pi$ where $P$ and $V$ are virtual $\bar{q} q$ objects having the quantum numbers of ground state pseudoscalar and vector mesons respectively. Such processes often arise in meson exchange models of electroproduction of mesons from hadronic targets. Our model of the underlying quark-gluon dynamics is used to investigate the extent to which the virtuality of $V$ and $P$ influences the corresponding form factors that should be employed in meson exchange models, given that the employed $t$-channel propagator is of the standard point meson type.

Here we model the hadronic coupling to $V$ or $P$ as generated by a point $\bar{q} \gamma_{\mu} q$ or $\bar{q} \gamma_{5} q$ vertex accompanied by a standard phenomenological meson-hadron form factor. The physics of interaction and propagation of $V$ or $P$ towards the $\gamma^{*} \pi$ or $\gamma^{*} \rho$ transition currents is implemented by solution of the ladder BSE for the corresponding dressed versions of the $\bar{q} \gamma_{\mu} q$ or $\bar{q} \gamma_{5} q$ vertices. We contrast the results obtained this way with those from the meson exchange picture in which the $\gamma^{*} \pi \rightarrow \rho$ and $\gamma^{*} \rho \rightarrow \pi$ form factors are paired with the corresponding point meson propagator. In this way we obtain some insight into the domain of applicability of the meson ex- 
change mechanism for these processes.

We find that near the photon point, the dependence of the amplitude for $\gamma^{*} V \rightarrow \pi$ upon the spacelike virtuality of $V$ is well described by the meson exchange picture out to at least $5 \mathrm{GeV}^{2}$, which is the limit of our examination. As we have pointed out, there is indirect information that the agreement will extend to asymptotic spacelike momenta. However when both $\gamma^{*}$ and $V$ are more spacelike than about $1 \mathrm{GeV}^{2}$, the point meson propagator for $V$ overestimates the falloff with virtuality of $V$ by at least $50 \%$ and the discrepancy increases with virtuality. This means that the $\gamma^{*} V \pi$ form factor to be used in effective point meson exchange models must have a dependence upon virtuality of $V$ that compensates for the inadequacy of the point meson propagator. Similar conclusions are drawn for the $\gamma^{*} P \rightarrow \rho$ process.

Rather than model the coupling of the $\bar{q} q$ correlation to a nucleon source by a $\bar{q} q$ dressed vertex seeded by a point vertex and a phenomenological meson-nucleon form fac- tor, a more exact treatment would require a quark-gluon description of the nucleon transition current. It is a difficult task to combine such a description with the meson transition form factors considered here. Some progress in development of the required techniques within a DSE approach can be found in Ref. 433].

\section{Acknowledgments}

We are grateful to T.S.H. Lee for a number of helpful discussions and questions that led to some of the present investigations. This work was funded by the National Science Foundation under Grant Nos. PHY9722429 and PHY-0071361 and by the Department of Energy under Grant Nos. DE-FG02-96ER40947 and DEFG02-97ER41048; it benefitted from the resources of the National Energy Research Scientific Computing Center.
[1] M. Gockeler et al., Phys. Rev. D53, 2317 (1996), heplat/9508004.

[2] M. Gockeler, R. Horsley, D. Pleiter, P. E. L. Rakow, and G. Schierholz (2001), hep-ph/0108105.

[3] U. Vogl and W. Weise, Prog. Part. Nucl. Phys. 27, 195 (1991).

[4] R. T. Cahill and S. M. Gunner, Fizika B7, 171 (1998), hep-ph/9812491.

[5] P. C. Tandy, Prog. Part. Nucl. Phys. 39, 117 (1997), nucl-th/9705018.

[6] H. Weigel, E. Ruiz Arriola, and L. P. Gamberg, Nucl. Phys. B560, 383 (1999), hep-ph/9905329.

[7] E. Ruiz Arriola (2001), hep-ph/0107087.

[8] C. D. Roberts and S. M. Schmidt, Prog. Part. Nucl. Phys. 45S1, 1 (2000), nucl-th/0005064.

[9] R. Alkofer and L. von Smekal, Phys. Rept. 353, 281 (2001), hep-ph/0007355.

[10] P. Maris and C. D. Roberts, Phys. Rev. C56, 3369 (1997), nucl-th/9708029.

[11] P. Maris and P. C. Tandy, Phys. Rev. C60, 055214 (1999), nucl-th/9905056.

[12] M. B. Hecht, C. D. Roberts, and S. M. Schmidt, Phys. Rev. C63, 025213 (2001), nucl-th/0008049.

[13] P. Maris and P. C. Tandy, Phys. Rev. C61, 045202 (2000), nucl-th/9910033.

[14] T. Sato and T. S. H. Lee, Phys. Rev. C63, 055201 (2001), nucl-th/0010025.

[15] P. Maris and P. C. Tandy, Phys. Rev. C62, 055204 (2000), nucl-th/0005015.

[16] P. Maris, C. D. Roberts, and P. C. Tandy, Phys. Lett. B420, 267 (1998), nucl-th/9707003.

[17] A. Bender, C. D. Roberts, and L. Von Smekal, Phys. Lett. B380, 7 (1996), nucl-th/9602012.

[18] F. T. Hawes, P. Maris, and C. D. Roberts, Phys. Lett. B440, 353 (1998), nucl-th/9807056.

[19] P. Maris (2000), nucl-th/0009064.

[20] P. C. Tandy (2001), nucl-th/0106031.

[21] J. I. Skullerud and A. G. Williams, Phys. Rev. D63, 054508 (2001), hep-lat/0007028.
[22] J. Skullerud, D. B. Leinweber, and A. G. Williams, Phys. Rev. D64, 074508 (2001), hep-lat/0102013.

[23] D. E. Groom et al. (Particle Data Group), Eur. Phys. J. C15, 1 (2000).

[24] J. Volmer et al. (The Jefferson Lab F(pi) Collaboration), Phys. Rev. Lett. 86, 1713 (2001), nucl-ex/0010009.

[25] P. Maris, Nucl. Phys. Proc. Suppl. 90, 127 (2000), nuclth/0008048.

[26] P. Maris and P. C. Tandy (2001), nucl-th/0109035.

[27] D. W. Jarecke, P. Maris, and P. C. Tandy (2002), in progress, KSUCNR-101-01.

[28] M. R. Frank, K. L. Mitchell, C. D. Roberts, and P. C. Tandy, Phys. Lett. B359, 17 (1995), hep-ph/9412219.

[29] C. D. Roberts, Nucl. Phys. A605, 475 (1996), hep$\mathrm{ph} / 9408233$.

[30] P. Maris and C. D. Roberts, Phys. Rev. C58, 3659 (1998), nucl-th/9804062.

[31] H. J. Behrend et al. (CELLO), Z. Phys. C49, 401 (1991).

[32] J. Gronberg et al. (CLEO), Phys. Rev. D57, 33 (1998), hep-ex/9707031.

[33] G. P. Lepage and S. J. Brodsky, Phys. Rev. D22, 2157 (1980).

[34] I. V. Anikin, A. E. Dorokhov, and L. Tomio, Phys. Lett. B475, 361 (2000), hep-ph/9909368.

[35] M. K. Chase, Nucl. Phys. B167, 125 (1980).

[36] D. Klabucar and D. Kekez, Fizika B8, 303 (1999), hepph/9905251.

[37] C. D. Roberts, Fizika B8, 285 (1999), nucl-th/9901091.

[38] P. C. Tandy, Fizika B8, 295 (1999), hep-ph/9902459.

[39] V. A. Viktorov et al., Pisma Zh. Eksp. Teor. Fiz. 33, 239 (1981).

[40] P. C. Tandy, Prog. Part. Nucl. Phys. 36, 97 (1996), nuclth/9605029.

[41] J. W. Van Orden, N. Devine, and F. Gross, Phys. Rev. Lett. 75, 4369 (1995).

[42] J. W. Van Orden (1999), private communication.

[43] P. Bicudo et al. (2001), hep-ph/0112015. 